\title{
Verb argument structure effects on tense: evidence from aphasia in Greek
}

Fani Nasika

School of Allied Health Professions, Canterbury Christ Church University, UK

School of Health, Aegean College, Greece

https://doi.org/10.36505/ExLing-2017/08/0019/000321

\begin{abstract}
This paper aims to present the findings of a study on tense in Greek-speaking individuals and whether it is affected by verb argument structure. Research evidence has shown that tense is impaired in aphasia but most of the studies concerned testing tense in isolation and not in dynamic contexts and in interaction with other factors. In this study, non-fluent and non-brain-damaged participants were tested on a twochoice sentence completion and a grammaticality judgment task. The results showed a significant difference in tense performance between the two groups but no overall significant effects of verb argument structure. However, there was individual variation within the non-fluent group which could be explained by the severity of aphasia, a processing or a tense deficit.
\end{abstract}

Key words: tense, verb argument structure, aphasia, Greek

\section{Theoretical Background}

The aim of the present study was to examine verb argument structure effects on tense in individuals with aphasia who are also native Greek speakers. Aphasia is an acquired language disorder caused by brain lesions in the language-dominant hemisphere which is usually the left hemisphere. A widely accepted aphasia classification system distinguishes two broad aphasia categories: non-fluent and fluent aphasia (Benson 1967). Non-fluent aphasia and, in particular, Broca's aphasia, is commonly related to a verb and tense deficit.

Tense is a grammatical feature and as such it denotes the time when an action has occurred. In languages such as English and Greek, tense is expressed through affixation, by combining a suffix with a verb stem. In these languages, Tense can be either [+PAST] or [-PAST]. Tense has been repeatedly found to be impaired in Broca's aphasia as in Kok et al. (2006), who observed that tense inflection was impaired in Dutch agrammatic individuals while similar diifuclties have been shown for Greek, too (Tsapkini, Jarema \& Kehayia 2000; Varlokosta et al. 2006).

On the other hand, verb argument structure seems to affect verb production and processing in aphasia. Verb argument structure involves the subject of the verb and its complements. More precisely, a verb's argument structure may involve one argument, two arguments or three arguments,

ExLing 2017: Proceedings of 8th Tutorial and Research Workshop on Experimental Linguistics, 19-22 June, Heraklion, Crete, Greece 
which may be optional or obligatory. Moreover, the argument structure of some verbs involves argument movement. These verbs form the unaccusative category which is distinct from the unergative category.

Verb argument structure has been shown to be an influencing factor in aphasia. More precisely, verb argument structure complexity has been reported to affect verb processing in aphasia (Shapiro et al. 1987). Moreover, it has been claimed that two-argument verbs are processed faster than threeargument verbs (Shapiro, Gordon, Hack \& Killakey 1993) while threeargument verbs seem more difficult to produce than two-argument verbs (Thomspon et al. 1997). Unaccusative verbs also seem more difficult to produce than unergative verbs (Thomspon 2003). The present study, thus, tested verb argument structure complexity effects on tense in Greekspeaking individuals with aphasia. The main hypotheses claimed that if verb argument structure complexity affects verb production and processing, then it might also be involved in the tense deficit often seen in aphasia.

\section{Methodology}

\section{Sample}

The study examined two groups of participants: 1) individuals with nonfluent aphasia and 2) non-brain-damaged individuals. The experimental group included four participants with non-fluent aphasia while the control group included six Greek native speakers. The participants in the control group had no neurological or sensory deficits. The participants in the nonfluent group were diagnosed based on the Greek standardized adaptation of the Boston Diagnostic Aphasia Examination (Papathanasiou et al. 2004).

\section{Material and procedure}

The testing procedure included two tasks. The first task was a two-choice sentence completion task and the second one was a grammaticality judgment task. The verbs included in these tasks were selected based on their high frequency, familiarity, and imageability. The first task consisted of 119 test items. The participants were presented with sentences which began with the time adverb yesterday in Greek but were missing their main verb. The participants had to complete the gap with one of two choices, with the verb either in the present or past tense. The grammaticality judgment task included 100 test sentences. The sentences were judged based on their verb argument structure or tense. 
Verb argument structure effects on tense: evidence from aphasia in Greek 75

\section{Results}

The data collected from this procedure was analysed with multiple regression analysis. In the two-choice sentence completion task, the nonfluent group scored $65.13 \%$ correctly while the control group scored $99.72 \%$. The two groups had a similar performance pattern regarding the number of arguments of the verbs. Performance was more accurate on verbs that had three optional arguments than two optional arguments, although this difference was not significant. In addition, the non-fluent group was more accurate when choosing the past tense for unergative verbs than for unaccusative verbs, but this difference was not significant either. Overall, in this task, the non-fluent group differed significantly from the control group in their ability to choose the past tense, irrespective of the argument structure of the verb. In the grammaticality judgment task, the non-fluent group's overall performance was lower than that of the control group $(70.5 \%$ vs. $97.5 \%$ ), with the first group showing a tendency to over-judge sentences as grammatical. This group, thus, showed a difficulty in judging ungrammatical sentences for their argument structure or tense. Correct performance varied across the two groups by the number of arguments of the sentences, with the non-fluent group judging two-argument structures more accurately than three-argument structures. Finally, the two groups differed significantly in their correct performance as far as grammatical and ungrammatical sentences were concerned.

\section{Conclusions}

Overall, this study has not found significant argument structure effects on tense in Greek aphasia, as in Koukoulioti and Stavrakaki (2014), while no significant difference was observed between unaccusative and unergative verbs. What was observed is the fact that the tense deficit in non-fluent aphasia is evident and does not concern only production. In addition, performance ranged within the non-fluent group regarding number of argument or argument structure complexity. The patterns seen comply more with the individualized profile of each participant and the level of the severity of their aphasia. In addition, processing parameters should also be taken into consideration since performance may have varied due to task demands or the processing abilities of each participant. Thus, the tense deficit seen in non-fluent aphasia should be interpreted in light of the severity of the aphasia, a processing deficit or tense specification difficulties so as to clarify clinical aspects for more effective clinical interventions. 


\section{References}

Benson, D. F. 1967. Fluency in aphasia: correlation with radioactive scan localization. Cortex Vol. 3 No 4, 373-394.

Kok, P., Kolk, H., Haverkort, M. 2006. Agrammatic sentence production: Is verb second impaired in Dutch? Brain and Language 96, 243-254.

Koukoulioti, V., Stavrakaki, S. 2014. Producing and inflecting verbs with different argument structure: Evidence from Greek aphasic speakers. Aphasiology 28 (11), 1320-1349.

Papathanasiou, I., Feidatsi, M., Katsantoni, M., Panagiotopoulou, E., Malefaki, S. 2004. The validation of Boston Diagnostic Aphasia Examination (BDAE-3) in Greek. Paper presented at the 26th World Congress of the International Association of Logopaedics and Phonetics, 29 August-2 September 2004, Brisbane, Australia.

Shapiro, L. P., Zurif, E., Grimshaw, J. 1987. Sentence processing and the mental representation of verbs. Cognition 27, 219-246.

Shapiro, L. P., Gordon, B., Hack, N., Killackey, J. 1993. Verb-Argument Structure Processing in Complex Sentences in Broca's and Wernicke's Aphasia. Brain and Language 45, 423-447.

Thompson, C. K. 2003. Unaccusative verb production in agrammatic aphasia: the argument structure complexity hypothesis. Journal of Neurolinguistics 16, 151167.

Thompson, C. K., Lange, K. L., Schneider, S. L., Shapiro, L. P. 1997. Agrammatic and non-brain-damaged subjects' verb and verb argument structure production. Aphasiology 11, 473-490.

aphasia: Evidence from agreement, tense and aspect. Aphasiology 20 (8), 723-743. 